\title{
Efeitos da abordagem osteopática na doença pulmonar obstrutiva crônica
}

\section{Effects of the osteopathic approach to chronic obstructive pulmonary disease}

\author{
Luciano Santos da Silva Filho ${ }^{1}$ (D) , Jean Michel Regis Mendes ${ }^{1}$ (D), Maurício Rocha Mendes ${ }^{2}$ (D) Cymara Pessoa Kuehner $^{3}$ (iD \\ 1. Pós-graduação em Osteopatia pelo Centro Universitário Christus (Unichristus), Fortaleza, CE, Brasil. 2. Pós-graduação em Osteopatia pelo Centro \\ Universitário Christus (Unichristus), Fortaleza, CE, Brasil. 2. Docente do curso de Osteopatia pelo Centro Universitário Christus (Unichristus), Fortaleza, CE, \\ Brasil. 3. Doutora em Ciências Médicas e Docente do curso de Osteopatia pelo Centro Universitário Christus (Unichristus), Fortaleza, CE, Brasil.
}

\begin{abstract}
Resumo
Objetivo: Realizar uma revisão integrativa sobre os efeitos da abordagem osteopática na doença pulmonar obstrutiva crônica. Metodos: Estudo seccional e documental, realizado por meio de revisão integrativa da literatura disponível nas bibliotecas virtuais de saúde: National Library of Medicine (PubMed), Literatura Latino-Americana e do Caribe em Ciências da Saúde (LILACS) e Physiotherapy Evidence Database (PEDro); no período de junho a outubro de 2019, através dos seguintes descritores em língua portuguesa e inglesa: Manipulação Osteopática, DPOC e Fisioterapia. Resultados: Foram encontrados 18 estudos potenciais inicialmente, dos quais 7 artigos foram considerados elegíveis para análise qualitativa. Conclusão: a utilização da Osteopatia como método coadjuvante apresenta evidências, embora incipientes, mas que já dão indícios dos seus efeitos na patologia. Dentre eles, estão: melhora da mecânica respiratória e da mobilidade diafragmática, aumento da capacidade de exercícios com diminuição da dispneia, além de efeitos nas variáveis pulmonares como CVF e VEF1, ainda não tão esclarecidos.
\end{abstract}

Palavras-chave: Manipulação Osteopática. DPOC. Fisioterapia.

\section{Abstract}

Objective: To conduct an integrative review on the effects of osteopathic approach on chronic obstructive pulmonary disease. Methods: Cross-sectional and documentary study conducted through an integrative review of literature available in virtual health libraries: National Library of Medicine (PubMed), Latin American and Caribbean Health Sciences Literature (LILACS), and Physiotherapy Evidence Database (PEDro); from June to October 2019, from the following descriptors in Portuguese and English: Osteopathic Manipulation, COPD and Physiotherapy. Results: Eighteen potential studies were initially found, of which 7 articles were considered eligible for qualitative analysis. Conclusion: The use of osteopathy as a supporting method has presented evidence, although incipient, but which already give evidence of its effects on the pathology. These include: improvement of respiratory mechanics and diaphragmatic mobility, increased exercise capacity with decreased dyspnea, and effects on pulmonary variables such as FVC and FEV1, which are still not so clear.

Keywords: Osteopathic Manipulation. COPD. Physicaltherapy.

\section{INTRODUÇÃO}

As doenças respiratórias, como pneumonia, Doença Pulmonar Obstrutiva Crônica (DPOC), bronquite e bronquiolite aguda, e asma, são responsáveis pelos maiores índices de internação hospitalar e óbitos, gerando altos custos para os serviços de saúde ${ }^{1}$. Segundo dados da Organização Mundial da Saúde (OMS), estima-se que 251 milhões de pessoas no mundo tenham o diagnóstico de DPOC, sendo esta a terceira principal causa de mortalidade no mundo².

As afecções respiratórias são enfermidades que acometem tanto as vias aéreas superiores como inferiores, que podem cursar para a cronicidade. As mais comuns estão a Asma, a Rinite Alérgica (RA) e a DPOC. No caso da DPOC, sua ocorrência está relacionada com a idade, podendo evoluir com a presença de comorbidades crônicas, como Hipertensão Arterial e Diabetes ${ }^{3}$.

A respeito da DPOC, esta consiste numa obstrução persistente do fluxo aéreo determinada por uma resposta inflamatória das vias aéreas e pulmões a agentes ou gases nocivos ${ }^{4}$. Sua progressão leva a uma significativa incapacidade, perda da produtividade e piora na qualidade de vida dos pacientes ${ }^{5}$ Apesar de seu caráter crônico, apresenta também episódios de agudização definidos com piora clínica de rápida instalação dos sintomas de dispneia e fadiga ${ }^{6}$.

Consideradas como terapias complementares ou integrativas, as técnicas osteopáticas representam um método sistemático de avaliação e tratamento das disfunções do sistema neuromusculoesquelético, com a finalidade de restabelecer o movimento fisiológico em áreas com restrição ou disfunção, promovendo o funcionamento adequado dos sistemas adjacentes $^{7,8}$. Nesse sentido, a Osteopatia é considerada uma abordagem que trata o sujeito como um ser único e integrado, que depende de inter-relações entre estrutura e função ${ }^{9}$.

Atualmente, as disfunções respiratórias são prioridade para os serviços de Saúde; entretanto, há uma discussão a respeito do acesso, bem como da resolutividade das práticas em saúde voltadas para essas alterações. Ressalta-se nos espaços de debates a necessidade de ampliação da cobertura dos serviços

Correspondente: Luciano Santos da Silva Filho. Centro Universitário Christus (Unichristus), Fortaleza, CE, Brasil. Email: lucianofilhofisio@gmail.com Conflito de interesse: Não há conflito de interesse por parte de qualquer um dos autores. 
em todos os níveis de atenção a saúde ${ }^{3}$.

Assim, pode-se observar que há estudos relatando os efeitos positivos das técnicas de terapia manual na cervicalgia, lombalgia, nas mudanças das propriedades viscoelásticas dos tecidos; porém, na literatura, são escassos os estudos sobre a utilização das técnicas da terapia manual, seja manipulativa, ou de mobilização na musculatura respiratória ${ }^{10}$. A partir disso, o estudo justifica-se pela necessidade de sistematização das pesquisas já realizadas sobre a temática, como também pode direcionar e incrementar as práticas dos fisioterapeutas no atendimento a pacientes com DPOC.

Esta pesquisa apresenta-se relevante, pois permite uma sistematização das técnicas de manipulação osteopáticas que tem efeitos no tratamento de desordens provenientes da DPOC, sendo uma prática complementar ao tratamento fisioterapêutico convencional. A partir disso, este estudo objetiva realizar uma revisão de literatura sobre os efeitos das técnicas de manipulação osteopáticas na DPOC.

\section{MÉTODOS}

Este estudo consiste numa revisão de literatura integrativa, considerada um método no campo da saúde que objetiva o direcionamento das práticas através da fundamentação teórica, assegurando, assim, uma assistência baseada em evidências científicas ${ }^{11}$.

Figura 1. Representação esquemática do processo de seleção dos artigos.
Para guiar a pesquisa, formulou-se a seguinte questão: Quais evidências científicas revelam os efeitos da abordagem osteopática na Doença Pulmonar Obstrutiva Crônica?

Na coleta, foram utilizadas as seguintes bases de dados: National Library of Medicine (PubMed), Literatura Latino-Americana e do Caribe em Ciências da Saúde (LILACS) e Physiotherapy Evidence Database (PEDro); no período de junho a outubro de 2019, a partir dos seguintes descritores em língua portuguesa e inglesa: Manipulação Osteopática, DPOC e Fisioterapia.

Para a pesquisa, foram utilizados os operadores boleanos AND e OR, sem restrição linguística. Assim, foram incluídos na pesquisa artigos publicados nos últimos dez anos, nos idiomas Português, Inglês e Espanhol, que apresentassem relevância para o tema pesquisado associado a uma metodologia válida e consistente.

\section{RESULTADOS E DISCUSSÕES}

A Através dos descritores Manipulação Osteopática AND DPOC AND Fisioterapia, foram encontrados 18 artigos. Após avaliação dos títulos e resumos, 10 trabalhos foram submetidos aos critérios de inclusão, destes foram excluídos os que não possuíam relação com o tema; os que estavam fora do período de tempo previsto; aqueles com textos incompletos e resumos, restando assim, sete trabalhos, conforme demonstrado na Figura 1. A respeito dos artigos selecionados, estes estão descritos na Tabela 1.

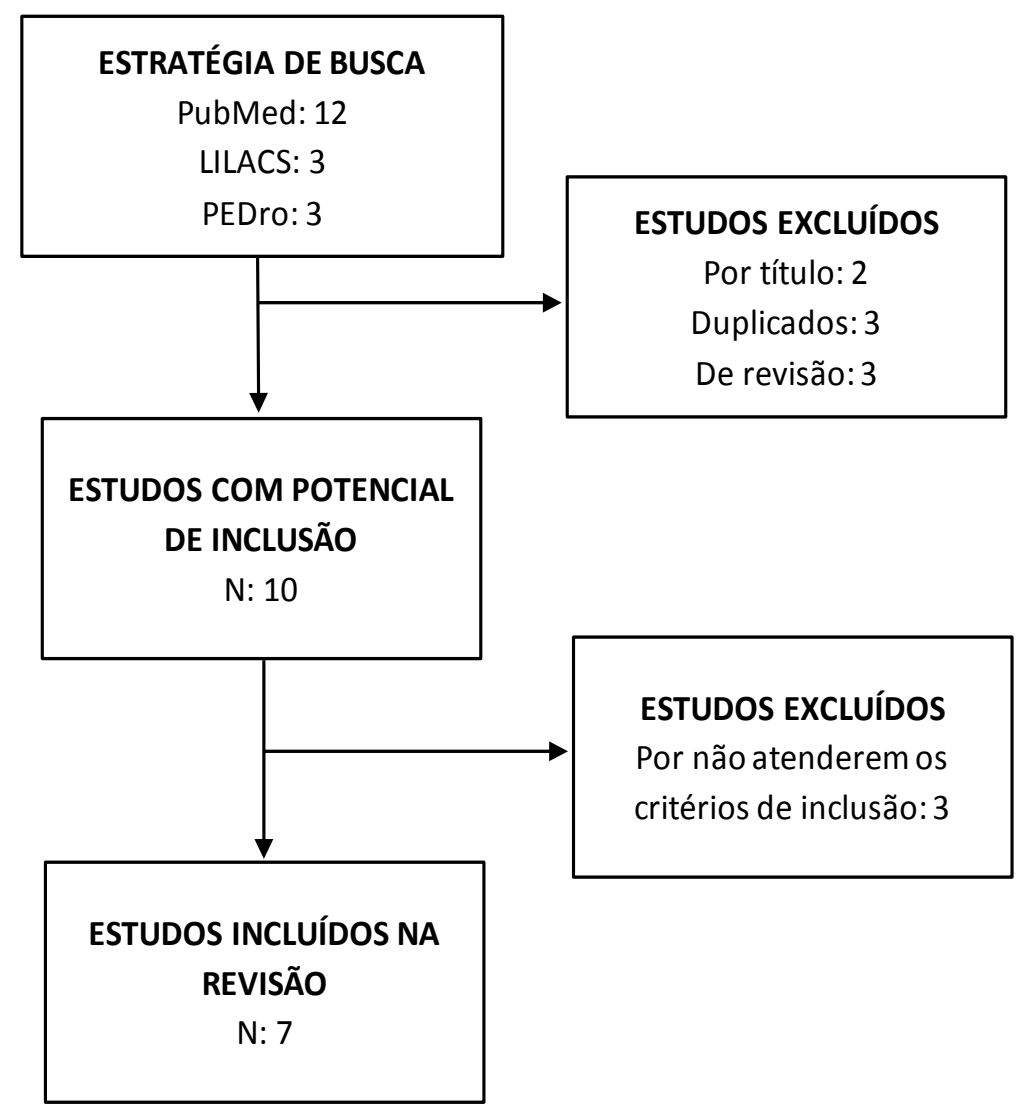




\begin{tabular}{llll}
\hline \multicolumn{1}{c}{ AUTOR / ANO } & TIPO DE ESTUDO & OBJETIVO & RESULTADOS \\
\hline BUSCEMI ${ }^{12}$ & Estudo piloto & Demonstrar que as técnicas Os pacientes do grupo intervenção \\
$(2019)$ & randomizado & osteopáticas, combinadas ao obtiveram melhores resultados \\
& & tratamento farmacológico, podem nos testes de espirometria: CVF \\
& & ser eficazes no tratamento dos $(p<0,5411), V E F 1$ total $(p<0,5061) ;$ \\
& sintomas da DPOC e melhorar teste de avaliação da DPOC \\
& o bem-estar e a vida diária dos ( $p<0,0005)$ e TC6 ( $p<0,0038)$, em \\
& pacientes afetados. & comparação ao grupo controle.
\end{tabular}

BARROS DE SÁ ${ }^{13}(2017) \quad$ Ensaio clínico randomizado

WADA $^{14}$

(2016)

ROCHA ${ }^{15}$

(2015)

ENGEL ${ }^{16}$

(2014)

ZANOTTI $^{17}$

(2012)
Ensaio clínico randomizado

Estudo unicêntrico randomizado

Ensaio clínico randomizado
Avaliar os efeitos imediatos do alongamento da musculatura respiratória na cinemática da parede torácica e na atividade eletromiográfica em pacientes com DPOC.

Avaliar os efeitos do treinamento aeróbico combinado com alongamento muscular respiratório na capacidade funcional de exercício e na cinemática toracoabdominal em pacientes com DPOC.

Avaliar os efeitos da TMLD na função respiratória de pessoas com DPOC.

Investigar o efeito da inclusão da terapia manual em um programa de reabilitação pulmonar para pacientes com DPOC.

Avaliar o efeito na capacidade de exercício, medida pelo TC6 e possíveis alterações na função pulmonar

CONCLUSÃO

A espirometria melhora, mas não é estatisticamente relevante. Os pacientes afetados pela DPOC moderada a grave podem se beneficiar fortemente da administração de tratamentos osteopáticos em associação com a terapia farmacológica tradicional.

Após uma única sessão, o grupo $\mathrm{O}$ estudo sugere $\mathrm{o}$ uso de intervenção aumentou o VCpt ( $p$ alongamento para melhorar a $=0,020)$ e VCpa $(p=0,043)$ e seus mobilidade da parede torácica da percentuais em relação à parede DPOC, com efeitos positivos na torácica, VCpt\% (p = 0,044) e mecânica do tórax, na distribuição VCpa\% $(p=0,022)$. de volume e na eletromiografia.

Após a intervenção, o GT Sugere-se que o treinamento apresentou melhora na aeróbico combinado com o contribuição abdominal, volume alongamento dos músculos compartimental, mobilidade e respiratórios aumenta a capacidade de exercício funcional capacidade de exercício funcional com diminuição da dispneia com diminuição da dispnéia quando comparado ao GC em pacientes com DPOC. Esses $(p<0,01)$. Além de uma diminuição efeitos estão associados ao do esforço muscular respiratório aumento da eficácia dos músculos necessário para obter o mesmo respiratórios e à participação do volume pulmonar em comparação compartimento abdominal. ao GC $(p<0,001)$.

TMLD

melhorou significativamente na mobilidade diafragmática ao longo dos tratamentos, com uma diferença entre os grupos na melhoria cumulativa de $18 \mathrm{~mm}$ (IC 95\% 8 a 28); no TC6 ao longo do curso do tratamento, com diferença entre grupos de 22 m (IC 95\% 11 a 32). A capacidade inspiratória estimada pela POE mostrou benefício cumulativo significativo de 330 ml (IC 95\% 100 a 560). Os efeitos em outros resultados foram não significativos ou pequenos.

Houve uma diferença significativa na CVF entre os três grupos em 24 semanas $(P=0.04)$.

Para o grupo $\mathrm{TM}+\mathrm{ME}+\mathrm{RP} \times \mathrm{RP}$ apenas o aumento foi de 0,40 l (IC: $0,02,0,79 ; \quad p=0,03)$. Não foram relatados eventos adversos maiores ou moderados após a administração de 131 intervenções com TM e 272 ME.

20 idosos em estágio severo de DPOC com disfunção somática no nível de OAA, C3-C4, T2-T9 e T12-L1, e disfunção de costelas na inspiração. Não houve efeitos adversos ou efeitos colaterais. Tratamento convencional e Osteopatiaforam bem tolerados.
O aumento da CVF é uma descoberta única. Embora os mecanismos subjacentes responsáveis por esse resultado ainda não sejam compreendidos, a explicação mais provável é o efeito sinérgico resultante da combinação de intervenções. Esses resultados apoiam a necessidade de um estudo clínico maior no uso de TM para DPOC.

A adição da Osteopatia ao Tratamento Convencional poderia aumentar a capacidade de exercício em pacientes com DPOC. 


\begin{tabular}{|c|c|c|c|c|}
\hline AUTOR / ANO & TIPO DE ESTUDO & OBJETIVO & RESULTADOS & CONCLUSÃO \\
\hline $\begin{array}{l}\text { NOLL }^{18} \\
(2009)\end{array}$ & Ensaio clínico & $\begin{array}{l}\text { Determinar os efeitos imediatos } \\
\text { de } 4 \text { técnicas osteopáticas nas } \\
\text { medidas da função pulmonar em } \\
\text { pessoas com DPOC. }\end{array}$ & $\begin{array}{l}\text { A TBLT com ativação aumentou } \\
\text { o VR pós-tratamento em } \\
\text { comparação à linha de base } \\
(\mathrm{d}=0,30) \text {, e a TBLT sem ativação } \\
\text { não }(d=0,12) \text {. A TEC foi associada } \\
\text { a uma diminuição pós-tratamento } \\
\text { em relação ao valor basal no } \\
\text { FEFmáx ( } d=0,55) \text { e VVM ( } d=0,75) \text {. } \\
\text { A liberação miofascial foi associada } \\
\text { a uma diminuição pós-tratamento } \\
\text { da linha de base no VEF1 (d=0,53), } \\
\text { FEF25-75\% ( } d=0,47) \text {, FEFmáx } \\
\text { ( } d=0,60), M V V(d=0,53) \text { e CVL ( } d \\
=0,56) .\end{array}$ & $\begin{array}{l}\text { O componente de ativação da TBLT } \\
\text { aumentou o VR em pessoas com } \\
\text { DPOC. Os efeitos colaterais foram } \\
\text { relativamente leves e transitórios } \\
\text { e foram relacionados à dor na } \\
\text { parede torácica pós-tratamento. } \\
\text { As técnicas pioraram levemente } \\
\text { as medidas da função pulmonar } \\
\text { imediatamente após o tratamento } \\
\text { em relação às medidas da linha } \\
\text { de base. Entretanto os sujeitos } \\
\text { relataram subjetivamente que se } \\
\text { beneficiaram das manobras. }\end{array}$ \\
\hline
\end{tabular}

Fonte: Dados da pesquisa, 2019.

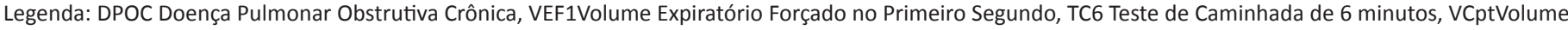

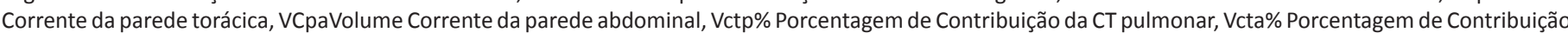

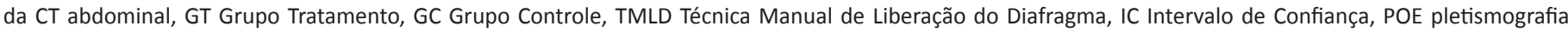

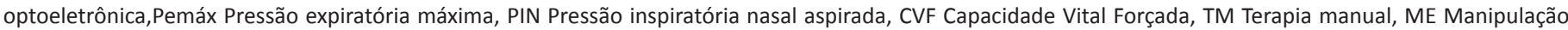

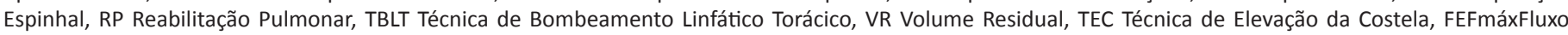
expiratório máximo forçado, VVM ventilação voluntária máxima, FEF25-75\% Fluxo expiratório forçado médio, CVL Capacidade Vital Lenta.

Segundo Santos et $a l .{ }^{8}$, a musculatura respiratória por estar sempre ativa e participar na manutenção do tórax, apresenta maior número de fibras tônicas, com tendência a retração, limitando a amplitude torácica, sugerindo menor oferta de oxigênio celular. Existe, também, uma relação com a progressão da idade e as alterações da complacência pulmonar e do gradil costal, com diminuição da capacidade elástica e aumento da resistência ao fluxo aéreo, gerando maior aprisionamento de $\operatorname{ar}^{15}$.

Assim, a medida que evolui, a DPOC provoca danos ao parênquima pulmonar, levando a alterações estruturais, bem como ao colapso dinâmico das pequenas vias aéreas. Esta alteração causa aprisionamento aéreo e hiperinsuflação pulmonar com repercussões nas fibras musculares do diafragma, redução da zona de aposição e repercussões na parede torácica ${ }^{13}$.

Nessa perspectiva, a mobilização ou manipulação de tecidos capsuloligamentares, como as técnicas osteopáticas, podem melhorar a extensibilidade do tecido que apresenta restrição, bem como ativar os mecanorreceptores articulares da região estimulada. Na parede torácica, essas técnicas tem o potencial de reverter algumas alterações tixotrópicas dos músculos respiratórios, provocando alívio na restrição articular da parede torácica e reduzindo, num curto prazo, a rigidez torácica ${ }^{8,16}$.

Buscemi et al. ${ }^{12}$ ressalta, ainda, que a abordagem osteopática considera tanto as alterações na mecânica respiratória quanto a anatomia do paciente, visando aumentar a mobilidade da caixa torácica, bem como estimular os processos de autocura do corpo humano.

É sabido que apenas uma sessão de alongamento muscular pode promover aumento da extensibilidade de tecidos moles, como visto no ensaio clínico randomizado de Barros de Sá et $a l{ }^{13}$. Na pesquisa, o grupo intervenção (15 sujeitos) teve aumento do VCpt $(p=0,020)$ e VCpa $(p=0,043)$ após uma sessão de alongamento, sugerindo uma melhora da mobilidade torácica com efeitos positivos na mecânica da parede torácica.

Corroborando o estudo, Rocha et al. ${ }^{15}$ apresenta a Técnica Manual de Liberação do Diafragma como intervenção para grupo de idosos com diagnóstico de DPOC estável. A técnica consiste na elevação das costelas inferiores associada aos ciclos respiratórios do paciente. Na pesquisa, a utilização da técnica em seis sessões apresentou melhora significativa da mobilidade diafragmática ao longo das sessões, com diferença cumulativa de $18 \mathrm{~mm}$ (IC 95\% 8 a 28) entre o grupo controle e intervenção.

A DPOC é caracterizada, principalmente, por fraqueza muscular dos músculos periféricos e respiratórios. Esse fenômeno leva ao aumento da dispneia, reduzida tolerância aos exercícios e limitações nas atividades de vida diária, prejudicando, assim, sua qualidade de vida e autonomia ${ }^{19}$.

Nessa perspectiva, Wada et al. ${ }^{14}$ realizou um ensaio clínico randomizado com 28 pacientes, divididos em Grupo Tratamento (GT) e Grupo Controle (GC). Antes do treinamento aeróbico, o GT era submetido ao stretching de escaleno, esterno cleidomastóideo, trapézio, peitorais maior e menor, intercostal, serrátil anterior e reto abdominal por três séries em três ciclos, com um minuto de relaxamento. Já o GC realizou alongamento ativo de flexores e extensores de punho e tornozelo por um minuto com mais um de descanso. Ambos os grupos realizaram treinamento aeróbico em esteira por 30 minutos, com velocidade média definida e percepção de esforço intenso.

No estudo, após 24 sessões, o GT apresentou melhora na contribuição abdominal, volume compartimental, mobilidade e capacidade de exercício funcional com diminuição da dispneia quando comparado ao $G C(p<0,01)$. E diminuição do esforço 
muscular necessário para obter o mesmo volume pulmonar em comparação ao GC $(p<0,001)^{14}$.

Em estudo anterior, Zanotti et al. ${ }^{17,20}$ idosos submetidos a um programa de reabilitação pulmonar (treinamento físico, apoio educacional e aconselhamento nutricional e psicológico), sendo que a metade da amostra recebeu manipulação suave (tratamento falso de osteopatia) e a outra submetida a um exame osteopático (anamnese; exame físico de saída torácica, coluna vertebral, caixa torácica, diafragma torácico e pélvico, e tentório cerebelar; e avaliação crânio-sacral), em seguida, receberam tratamento osteopático manipulativo.

Todos os sujeitos foram avaliados e reavaliados através da espirometria e do TC6. Após quatro semanas (40 sessões), o desfecho primário foi a alteração média do TC6 em ambos os grupos. O primeiro grupo ganhou $23,7 \pm 9,7 \mathrm{~m}$; enquanto o grupo que recebeu tratamento osteopático, teve um ganho adicional de $72,5 \pm 7,5 \mathrm{~m}(\mathrm{p}=0,01)$. A diferença entre os grupos ao final do estudo $(48,8 \mathrm{~m}$; IC $95 \%$ de 17 a $80,6 \mathrm{~m})$ foi significativa $(p=0,04)$. Quanto a função pulmonar, o grupo intervenção apresentou diminuição do VR de $4,4 \pm 1,51$ para $3,9 \pm 1,51(p=0,05)$ e melhora no VEF1, em que, no início, era de $0,99 \pm 0,41$ e aumentou $14 \%$ $(1,13 \pm 0,41)$ ao final ${ }^{17}$.

No que diz respeito à avaliação da função pulmonar, são utilizadas, principalmente, as seguintes variáveis: Volume Residual, Capacidade Pulmonar Total, Volume Expiratório Forçado no primeiro segundo e Capacidade Vital Forçada. Por exemplo, o declínio do VEF1 ainda tem-se relacionado com a progressão da doença. Todavia, sabe-se que a contribuição, independente das variáveis pulmonares para os desfechos, são menores quando correlacionadas juntas ${ }^{20,21}$.

Nessa perspectiva, Buscemi et al..$^{12}$ avaliou e reavaliou 32 indivíduos com diagnóstico de DPOC de moderada a grave através da espirometria, Teste de Avaliação da DPOC (COPD Assessment Test) e o TC6, aproximadamente duas vezes por mês, totalizando quatro avaliações. O grupo intervenção foi submetido a um protocolo osteopático que incluía técnicas de liberação miofascial para o tratamento do seio maxilar, ligamentos vertebropleurais, nervo frênico, costelas, pleura, pulmões, brônquios, músculo subclávio e ligamentos trapezóide e conóide, com o objetivo de mobilizar a caixa torácica e suas estruturas adjacentes de modo a melhorar suas funções.

Após quatro sessões, o grupo intervenção obteve resultados melhores nos testes de espirometria: CVF $(p<0,5411)$, VEF1 total $(p<0,5061)$; teste de avaliação da DPOC $(p<0,0005)$ e TC6 $(p<0,0038)$, em comparação ao grupo controle. Embora os resultados não sejam estatisticamente relevantes, a pesquisa mostrou que o paciente com DPOC pode se beneficiar do tratamento osteopático em associação com a terapia farmacológica tradicional ${ }^{12}$.

Corroborando com o estudo acima, Engel et al. ${ }^{16}$, submeteu 33 sujeitos a três grupos de intervenção: apenas Reabilitação
Pulmonar (RP); Terapia Manual (TM) e RP; Manipulação Espinhal (ME) com TM e RP. Após 24 sessões, o principal achado foi a diferença entre grupos para CVF ( $p=0.04)$. Quando comparado o último grupo com o primeiro, houve um aumento na CVF (0.40I, 98.33\% IC: $0.02,0.79 ; p=0.03)$.

No tocante a qual técnica osteopática é mais efetiva para a DPOC, Noll et al. ${ }^{18}$ propôs um estudo com 24 sujeitos submetidos a cinco sessões de tratamento; cada uma com uma técnica específica. De forma aleatória, com avaliação antes e 30 minutos após o tratamento, foram utilizadas as seguintes técnicas: Toque Mínimo (o médico realizava apenas ausculta pulmonar e cardíaca), Bombeamento Linfático Torácico com ativação e sem ativação, Elevação de Costelas e Liberação Miofascial. Cada técnica era realizada por cinco minutos, com exceção da Liberação Miofascial que durou de 5 a 10 minutos.

No estudo, observou-se que as quatro técnicas osteopáticas pioraram levemente as medidas da função pulmonar imediatamente após o tratamento em relação às medidas basais. A técnica de bombeamento com ativação aumentou o Volume Residual pós-tratamento em comparação à linha de base $(d=0,30)$, enquanto que a técnica sem ativação não $(d=0,12)$. A TEC foi associada a uma diminuição pós-tratamento em relação ao valor basal no FEFmáx $(d=0,55)$ e VVM $(d=0,75)$. A liberação miofascial foi associada a uma diminuição pós-tratamento da linha de base no VEF1 ( $d=0,53)$, FEF25-75\% $(d=0,47)$, FEFmáx $(d=0,60), M V V(d=0,53)$ e CVL $(d=0,56)$. Entretanto, mesmo com esses resultados nas medidas da função pulmonar, os sujeitos com DPOC participantes relataram subjetivamente que se beneficiaram da manipulação osteopáticas18.

\section{CONCLUSÃO}

Sabe-se que a DPOC cursa principalmente com a redução da tolerância aos esforços, sendo altamente incapacitante e afetando diretamente a qualidade de vida das pessoas que vivem com ela.

Nessa perspectiva, a utilização da Osteopatia como método coadjuvante tem apresentado evidências, embora incipientes, mas que já dão indícios dos seus efeitos na patologia. Dentre eles, estão: a melhora da mecânica respiratória e da mobilidade diafragmática, aumento da capacidade de exercícios com diminuição da dispneia, além de efeitos nas variáveis pulmonares como CVF e VEF1, ainda não tão esclarecidos.

Dessa forma, aponta-se como limitação para este estudo, o número limitado de estudos de alta qualidade neste tema para que conclusões mais específicas possam ser feitas.

É importante destacar que a Osteopatia tem ganhado cada vez mais espaço no meio científico, e que seus efeitos na DPOC, como em outras desordens respiratórias, carecem de investigações com maior rigor metodológico, através de estudos controlados, randomizados, com número amostral suficiente para gerar impacto na ciência. 


\section{REFERÊNCIAS}

1. Cruz DM, Ohara DG, Castro SS, Jamami M. Internações hospitalares, óbitos, custos com doenças respiratórias e sua relação com alterações climáticas no município de São Carlos - SP, Brasil. Medicina (Ribeirão Preto). 2016; 49(3): 248-57. doi: 10.11606/issn.2176-7262.v49i3p248-257.

2. McDonald VM, Osadnik CR, Gibson PG. Treatable traits in acute exacerbations of chronic airway diseases. Chron Respir Dis. 2019;16: 1479973119867954 doi:10.1177/1479973119867954.

3. Souza MC, Souza JN, Camelier FWR, Camelier AA. Produção do cuidado e a rede de atenção a pessoa com doença respiratória crônica: um estudo de revisão. Rev Pes Fisio. 2017; 7(4): 574-582.

4. Capeletti AM, Sousa ACS, Feitoza CL, Basso-Vanelli RP, Gomes ELFD, Costa D. Can a physical activity similar to activities of daily living cause dynamic hyperinflation and change the thoracoabdominal configuration in patients with chronic obstructive pulmonary disease?. Int J Chron Obstruct Pulmon Dis. 2019 Jun;14: 1281-1287. doi: https://doi.org/10.2147/COPD.S196223

5. Clarke S, Munro PE, Lee AL. The Role of Manual Therapy in Patients with COPD. Healthcare (Basel) 2019 Mar; 7(1): 21. doi: https://doi.org/10.3390/ healthcare7010021

6. Francalacci A, Pacheco N, Castro A, Carpes M. Avaliação do estado de saúde através da escala AQ 20 em pacientes internados com DPOC descompensado. Lif. St. 2017. 4(2):15-3. doi: https://doi.org/10.19141/2237-3756.lifestyle.v4.n2. p15-33.

7. Gurgel FFA, Câmara GLG, Oliveira Segundo VH, Knackfuss MI, Seabra EJG, Lima IPC. Reflexões sobre o emprego da osteopatia nas políticas públicas de saúde no Brasil. Fisio. Brasil. 2017; 18(3): 374-381. doi: http://dx.doi.org/10.33233/ fb.v18i3.1066.

8. Santos JJ, Santos MCA, Carli J, Rocha P, Previatti KEK. A Influência das técnicas de terapia manual osteopatica na função respiratória. Arq. Cienc. Saúde UNIPAR 2015; 19(3): 191-7. doi: https://doi.org/10.25110/arqsaude.v19i3.2015.2873.

9. Moschetta MS, Rodrigues ME, Rocha NS, Costa LMR. Aplicação do questionário QVWOQOL - Bref antes e após tratamento manipulativo osteopático. Mun Saúde. 2015; 39(4): 441-447.

10. Verhaeghe N, Schepers J, van Dun P, Annemans L. Osteopathic care for spinal complaints: A systematic literature review. PLoS ONE. Nov 2018; 13(11): e0206284. doi: https://doi.org/10.1371/journal.pone.0206284.

11. Silva HB, Soares JL. Análise da abordagem fisioterapêutica no tratamento da disfunção temporomandibular: revisão integrativa. Rev Elet Acer Saúde. 2018 Dez. Supl 19: e210-e210. doi: https://doi.org/10.25248/reas.e210.2019
12. Buscemi A, Pennisi V, Rapisarda A, Pennisi A, Coco M. Efficacy of osteopathic treatment in patients with stable moderate-to-severe chronic obstructive pulmonary disease: a randomized controlled pilot study. J Complement Integr Med. 2019 Aug; 17(1): pp. doi:10.1515/jcim-2018-0128

13. Barros de Sá R, Pessoa MF, Cavalcanti AGL, Campos SL, Amorim C, Dornelas de Andrade A. Immediate effects of respiratory muscle stretching on chest wall kinematics and electromyography in COPD patients. Respir Physiol Neurobiol. 2017 Aug; 242: 1-7. doi: 10.1016/j.resp.2017.03.002.

14. Wada JT, Borges-Santos E, Porras DC, Paisani DM, Cukier A, Lunardi AC, et al. Effects of aerobic training combined with respiratory muscle stretching on the functional exercise capacity and thoracoabdominal kinematics in patients with COPD: a randomized and controlled trial. Int J Chron Obstruct Pulmon Dis. 2016 Oct; 11: 2691-2700. doi:10.2147/COPD.S114548.

15. Rocha T, Souza H, Brandão DC, Rattes C, Ribeiro L, Campos SL, et al: The manual diaphragm release technique improves diaphragmatic mobility, inspiratory capacity and exercise capacity in people with chronic obstructive pulmonary disease: a randomised trial. J Physiother. 2015 Oct; 61(4): 182-189 doi: 10.1016/j.jphys.2015.08.009.

16. Engel RM, Gonski P, Beath K, Vemulpad S. Medium term effects of including manual therapy in a pulmonary rehabilitation program for chronic obstructive pulmonary disease (COPD): a randomized controlled pilot trial. J Man Manip Ther. 2016 May; 24(2): 80-89. doi:10.1179/2042618614Y.0000000074.

17. Zanotti E, Berardinelli P, Bizzarri C, Civardi A, Manstretta A, Rossetti S, Fracchia C. Osteopathic manipulative treatment effectiveness in severe chronic obstructive pulmonary disease: a pilot study. Complement Ther Med. 2012 FebApr; 20(1-2): 16-22. doi: 10.1016/j.ctim.2011.10.008.

18. Noll, DR, Johnson, JC, Baer, RW, Snider, EJ. The immediate effect of individual manipulation techniques on pulmonary function measures in persons with chronic obstructive pulmonary disease. Osteopath Med Prim Care. 2009 Oct; 3: 9. doi: 10.1186/1750-4732-3-9.

19. Almeida JTS, Schneider LF. A Importância da Atuação Fisioterapêutica para manter a Qualidade de Vida dos Pacientes com Doença Pulmonar Obstrutiva Crônica - DPOC. Rev Cient Fac Educ e Meio Ambient. 2019;10(1):168-77. doi: https://doi.org/10.31072/rcf.v10iedesp.795

20. Chuang ML, Lin IF. Investigating the relationships among lung function variables in chronic obstructive pulmonary disease in men. Peer J. 2019 Oct; 7 : e7829. doi:10.7717/peerj.7829.

21. Cavalheri V, Mantoani LC, Camillo CA, Pitta F. Correlações entre o índice BODE e variáveis máximas de esforço em pacientes com DPOC. ASSOBRAFIR Ciênc. 2016 Abr; 7(1):13-21.

\section{Como citar este artigo/How to cite this article:}

Silva LS Filho, Mendes JMR, Mendes MR, Kuehner CP. Efeitos da abordagem osteopática na doença pulmonar obstrutiva crônica. J Health Biol Sci. 2021; 9(1):1-6. 